\title{
Stainless Steel Microfibers for Strain-Sensing Smart Clay Bricks
}

\author{
Antonella D’Alessandro $\mathbb{D}$, Andrea Meoni, and Filippo Ubertini $\mathbb{D}$ \\ Department of Civil and Environmental Engineering, Via G. Duranti 93, 06125 Perugia, Italy \\ Correspondence should be addressed to Antonella D’Alessandro; antonella.dalessandro@unipg.it
}

Received 13 February 2018; Accepted 8 July 2018; Published 5 August 2018

Academic Editor: Fanli Meng

Copyright ( 2018 Antonella D’Alessandro et al. This is an open access article distributed under the Creative Commons Attribution License, which permits unrestricted use, distribution, and reproduction in any medium, provided the original work is properly cited.

\begin{abstract}
Life cycle monitoring of structural health of civil constructions is crucial to guarantee users' safety. An optimal structural health monitoring system allows to automatically detect, locate, and quantify any damage in structural elements, thus anticipating major risks of local or global failures. Critical issues affecting traditional monitoring systems are sensors' placement, hardware durability, and long-term reliability of the measurements. Indeed, sensors' deployment is crucial for an effective investigation of the static and dynamic characteristics of the structural system, whereby durability and long-term stability of sensing systems are necessary for long-term monitoring. A very attractive solution to some of these challenges is developing sensors made of the same, or similar, material of the structure being monitored, allowing a spatially distributed and long-term reliable monitoring system, by the use of self-sensing construction materials. Within this context, the authors have recently proposed new "smart clay bricks" that are strain-sensing clay bricks aimed at embedding intelligent monitoring capabilities within structural masonry buildings. While previous work focused on smart bricks doped with titanium dioxide and using embedded point electrodes, this work proposes an enhanced version of smart bricks based on the addition of conductive micro stainless steel fibers that possess higher electrical conductivity and a more suitable fiber-like aspect ratio for the intended application, as well as plate copper electrodes deployed on top and bottom surfaces of the bricks. The paper thus presents preparation and experimental characterization of the new smart bricks. The influence of different amounts of fibers is investigated, allowing the identification of their optimal content to maximize the gauge factor of the bricks. Both electrical and electromechanical experimental tests were performed. Overall, the presented results demonstrate that the new smart bricks proposed in this paper possess enhanced strain-sensing capabilities and could be effectively utilized as sensors within structural masonry buildings.
\end{abstract}

\section{Introduction}

Structural health monitoring (SHM) of buildings is a very attractive technology for enhancing safety of users and occupants [1-3]. Among different construction types, traditional masonry structures, including historic and monumental buildings, entail the greatest monitoring challenges due to the peculiar heterogeneity and complexity of their components, to material nonlinearity and to the possible activation of both local and global failure mechanisms. Traditional sensing systems for SHM are made of materials that are very different from those of the monitored constructions. Furthermore, they are usually applied on the external surface of the elements and have durability drawbacks. The high cost of such sensing systems is also a quite often limiting issue. In the last few years, much scientific attention has been devoted to smart construction materials exhibiting self-sensing capabilities [4-6]. Nevertheless, only a few of those works have been focused on smart materials suitable for structural applications, with the majority of the contributions devoted to cement-based materials and concretes [7-9]. The sensing ability of smart materials is typically obtained by doping traditional matrices with conductive nano- or microfillers. Most of the researches concern carbon-based inclusions, such as carbon nanotubes and carbon fibers [10]. The authors have studied the behavior of cementitious materials with carbon additions with a specific attention to dynamic strain sensing [11] and recently presented a new structural multifunctional 


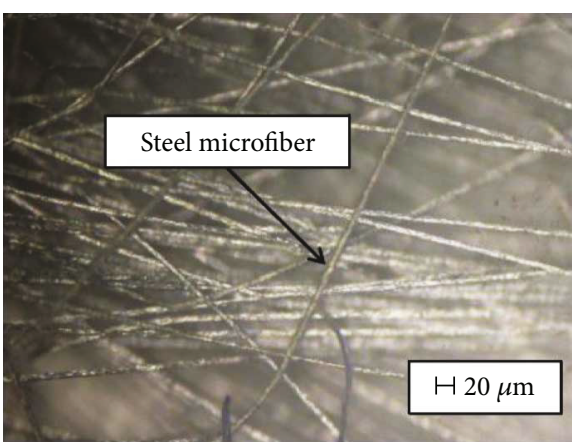

(a)

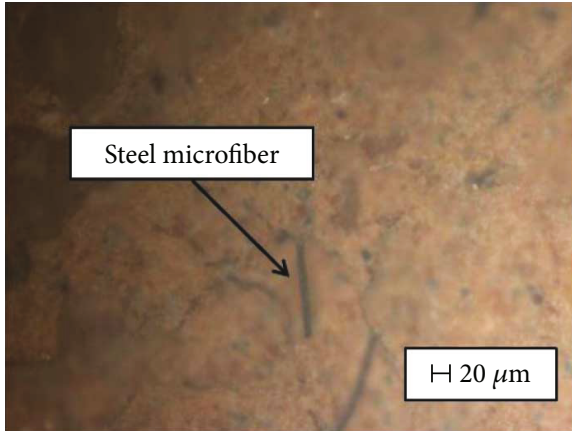

(b)

FIgURE 1: Micrographs of (a) stainless steel microfibers used as conductive filler for smart clay bricks and (b) a fragment of burned brick doped with stainless steel microfibers.

clay-based brick termed "smart brick" [12] with piezoresistive properties. Compared to a previous work, with this paper the authors propose a new fabrication methodology for smart bricks using ultrathin stainless steel microfibers (diameter of $12 \mu$ ) with a high level of conductivity and thermal resistance as reinforcement. It is also shown that plate electrodes made of copper to be attached on the top and the bottom surfaces of the bricks are more effective than previously adopted embedded wire stainless steel electrodes. After defining the new smart bricks and their fabrication procedure, laboratory tests are described and obtained results are discussed in order to demonstrate the smart bricks' strain-sensing properties.

\section{Materials and Preparation of Smart Bricks with Stainless Steel Microfibers}

Novel clay bricks were manufactured with different amounts of conductive micro inclusions consisting of stainless steel fibers: from 0 to $1 \%$ with steps of $0.25 \%, 1.5$, and $2 \%$, with respect to the total weight of the fresh clay. The fibers, model R.STAT/S, had a diameter of $12 \mu$ and length of $5 \mathrm{~mm}$ and were made of a special alloy (AISI 316L with Fe $68 \%, \mathrm{Cr}$ $18 \%$, Ni 12\%, and Mo 2\%) keeping low electrical resistivity values, of the order of $60-80 \Omega / \mathrm{cm}$, even after exposure to high temperatures, such as those reached during baking of clay around $1000^{\circ} \mathrm{C}$. Figure 1 shows a micrograph of the stainless steel microfibers (Figure 1(a)) and the magnification of a fragment of brick doped with stainless steel microfibers (Figure 1(b)) obtained using an inverted microscope, model Nikon Epiphot 300. The pictures clearly show the dimensional characteristics of the metallic fibers utilized in the experimentation and the acceptable dispersion obtained through the mechanical addition to the clay matrix.

The performance of the fibers at high temperature was investigated through thermogravimetric tests. Small amounts of fibers, kept in closed cups (Figure 2), were heated in an oven up to $900^{\circ} \mathrm{C}$, which is the temperature reached by the bricks during their preparation. Four subsequent thermal cycles were performed at increasing maximum temperature, from 300 to $900^{\circ} \mathrm{C}$ (Figure 2(a)). The fibers did not show any weight loss (Figure 2(b)). Before and after any thermal cycle, the electrical resistance of the fibers was measured using a multimeter instrumented with two clamps: no relevant decrease was observed (Figure 2(c)). The thermogravimetric tests confirmed that the stainless steel fibers were valid candidates as inclusions for conductive clay bricks.

The samples fabricated for the electromechanical tests were prisms with a square base of $5 \mathrm{~cm}$ sides and height of $7 \mathrm{~cm}$. The preparation process is sketched in Figure 3. Fibers and fresh clay were mixed for 10 minutes through an electric mixer for consistent mixtures (Figures 3(a) and 3(b)). The doped clay was poured into oiled molds sprinkled with sand (Figures 3(c) and 3(d)). Four stainless steel wires were embedded symmetrically in the central axis of the greater side of the brick (Figure 3(e)). Then, the bricks were dried at a temperature of $90^{\circ} \mathrm{C}$ for four hours and then burned at $900^{\circ} \mathrm{C}$ (Figures 3(f) and 3(g)) for six hours. This procedure was the same adopted by the authors for the preparation of bricks with nanotitania described in [12]. Particles of titania had a spherical shape, so they did not possess a prevalent dimension as the stainless steel fibers. Their dispersion was successfully achieved through a mechanical mixer. Each brick sample was instrumented with two $2 \mathrm{~cm}$-long electrical strain gauges, with a gauge factor of 2.1, applied on opposite lateral sides. Figure 3 refers to the case of embedded point stainless steel electrodes. In this paper, also horizontal copper plates are considered. In this case, copper plates of $50 \mu$ thickness were cut one $\mathrm{cm}$ larger than two opposite sides of the samples and applied after baking in order to be used as external conductive electrodes (Figure 4).

\section{Methodology}

Both electrical (with no mechanical loading) and electromechanical (under mechanical loading) tests were carried out. The samples were placed horizontally along the larger dimension. The two-probe method was adopted to measure bricks' electrical properties. Two different configurations were implemented: the first using the embedded stainless steel electrodes placed at a distance of $4 \mathrm{~cm}$ and the second with two copper plates externally applied at a distance of $5 \mathrm{~cm}$. Figure 4 shows the sketch of both setups and the photographs of the samples instrumented for electrical and electromechanical tests. 

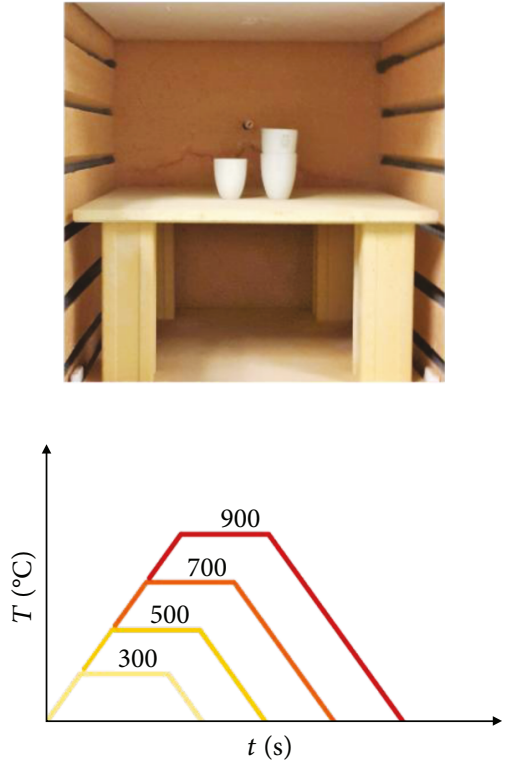

(a)

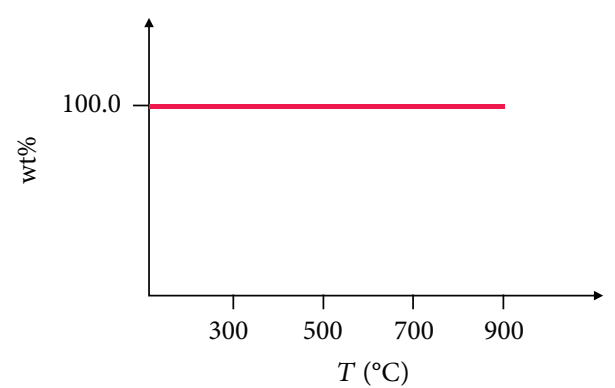

(b)

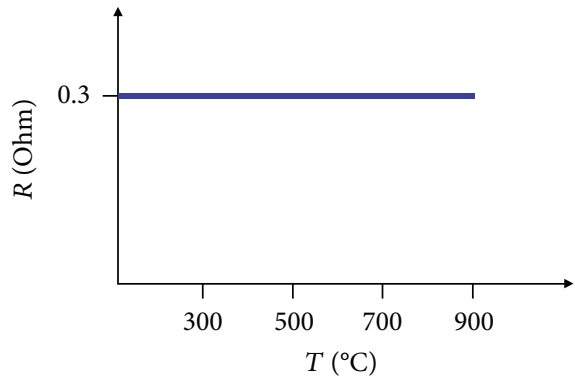

(c)

FIGURE 2: Thermogravimetric tests on stainless steel fibers: (a) waveforms of imposed thermal cycles; (b) residual weight after thermal cycles at increasing temperatures; (c) electrical resistance of the fibers after the thermal cycles at increasing temperatures.

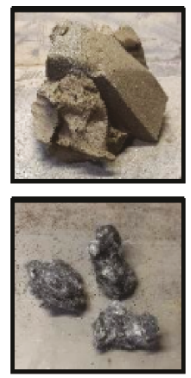

(a)

Preparation of clay and stainless steel fibers

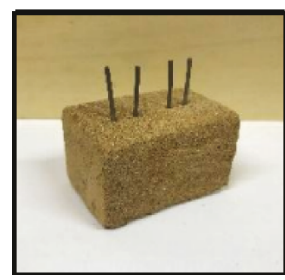

(h)

Smart brick

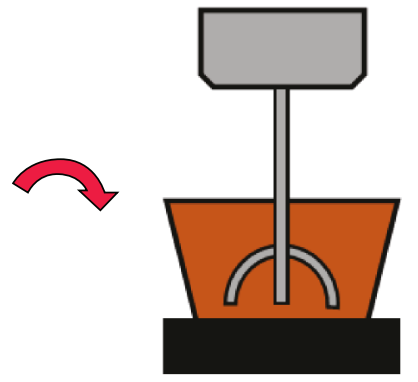

(b)

Mechanical mixing

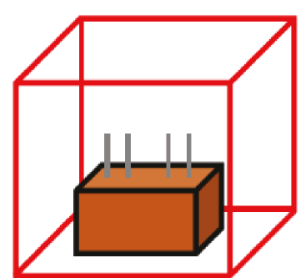

(g)

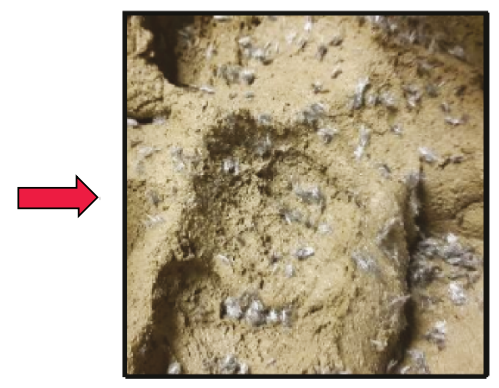

(c)

Doped clay

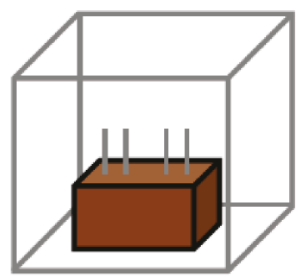

(f)

Drying in oven

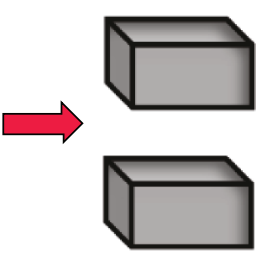

(d)

Moulding of briks
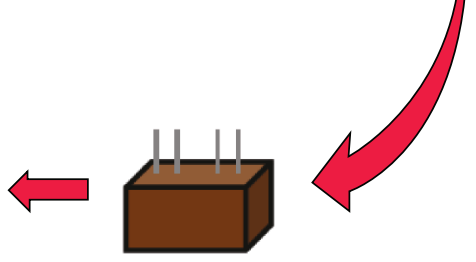

(e)

Embedding of electrodes

FIGURE 3: Preparation process of the clay brick samples with stainless steel microfibers.

3.1. Electrical Tests. Electrical tests were aimed at investigating the variation of electrical conductivity of the bricks with increasing amount of fillers and the effect of electrodes' configuration on the measurements. For this purpose, both embedded and external configurations of the electrodes were investigated. The tests were also aimed at verifying the repeatability of the measurements. The data acquisition system consisted of an NI PXIe-1073 chassis with a NI PXI4071 digital multimeter. A voltage square wave with an amplitude of $\pm 10 \mathrm{~V}(20 \mathrm{~V}$ peak-to-peak), $50 \%$ of duty cycle, and 
Sample of a smart brick
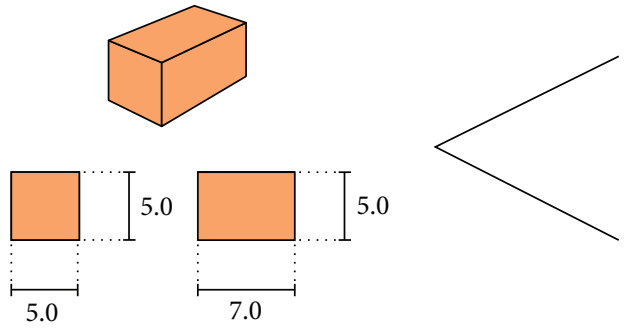

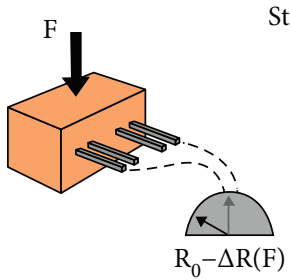

Stainless steel wire electrodes

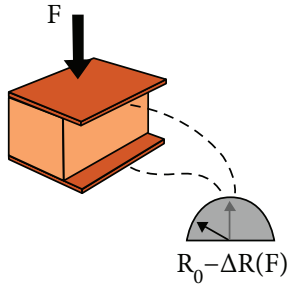

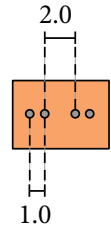

Copper plate electrodes

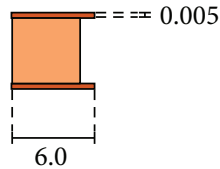

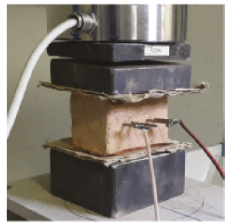

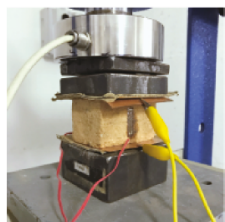

Figure 4: Bricks' dimensions and electrodes' configurations for electrical and electromechanical measurements (dimensions in $\mathrm{cm}$ ).
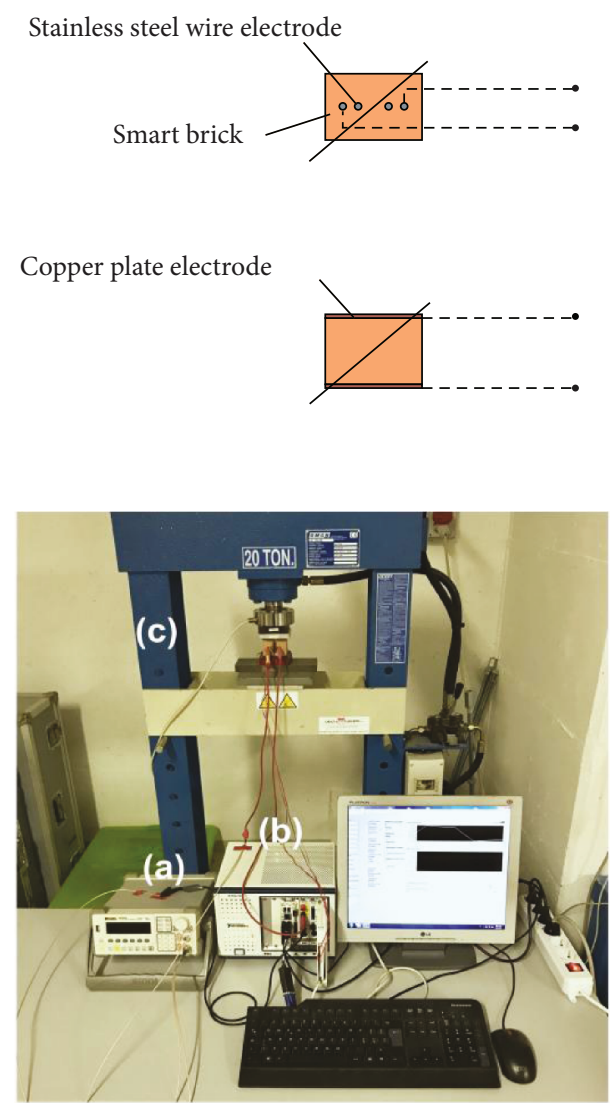

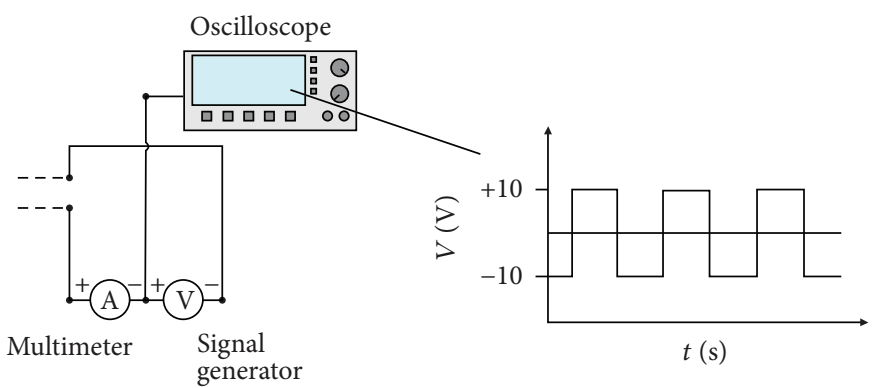

(a)
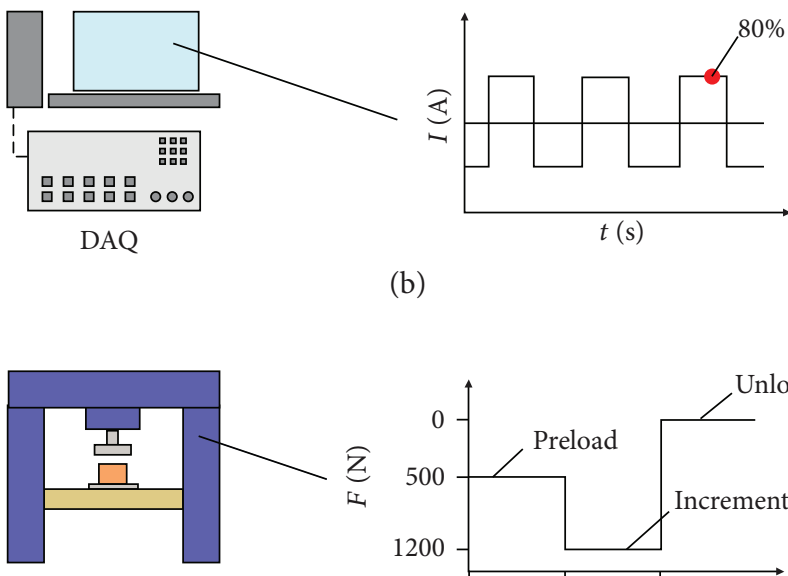

Press (b)

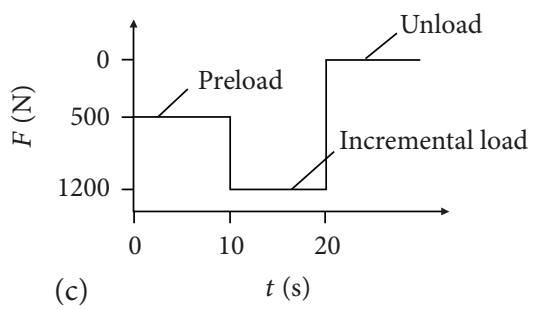

FIgURe 5: Photo evidence of the setup for the electrical and electromechanical tests with key components annotated (a) signal generator of the voltage square wave, (b) data acquisition system of the current (I), (c) applied square load.

frequency of $1 \mathrm{~Hz}$ (Figure 5(a)) was applied through a function generator, model Rigol DG1022, in order to produce the cycles of charge and discharge on the samples and eliminate the polarization effect. This effect is typical of dielectric materials, such as clay-based ones, and affects the electric measurements causing a time drift of the signal due to the generation of an electrical field opposite to the applied one. The adopted electrical configuration produces a low noise level, as demonstrated in previous works [13]. The current intensity measurements were taken in the positive portion of the biphasic signal, approximately at $80 \%$ of its constant part (Figure 5(b)), following the procedure defined in [13]. 


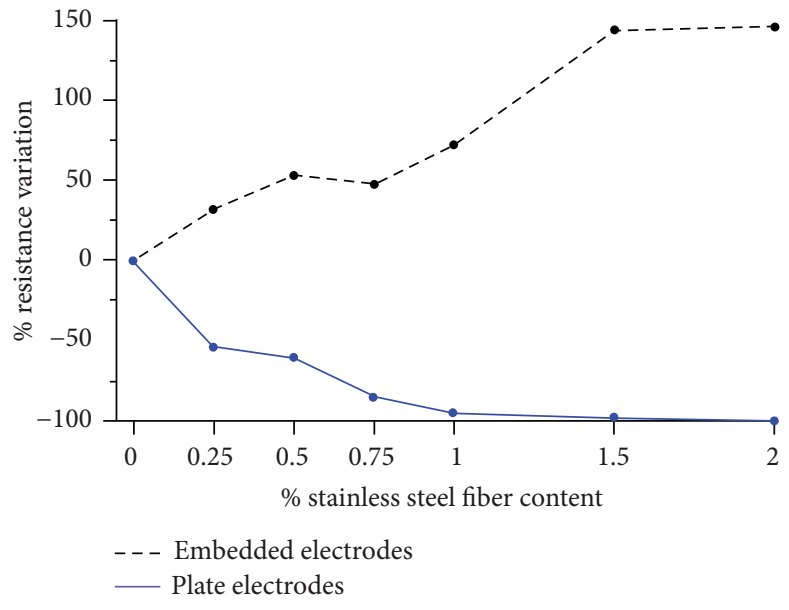

(a)

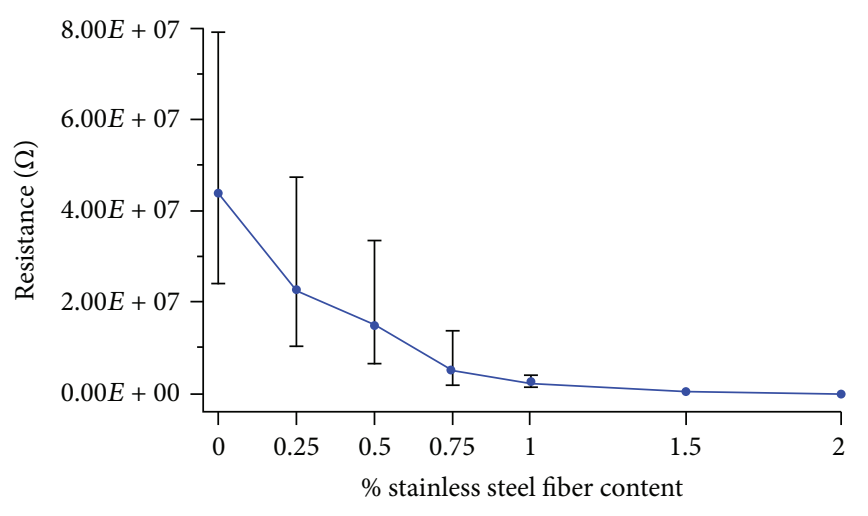

(b)

Figure 6: (a) Comparison between electrical resistance measured with embedded point electrodes and with copper plate electrodes for different stainless steel fibers contents; (b) average electrical resistance for different stainless steel fiber contents, with error bars representing the maximum and the minimum value of the electrical resistance variation obtained during electrical tests (10 repetitions of measurement).

The sampling rate in samples per second $(\mathrm{S} / \mathrm{s})$ was the same of the frequency of the applied voltage square wave.

The electrical resistance $R$ was calculated following Ohm's law:

$$
\left.R\right|_{t=\bar{t}}=\frac{V}{I_{t=\bar{t}}}
$$

where $V$ was the applied constant voltage and $I$ the measured current intensity, $\bar{t}$ being the specific time instant when the sample was taken.

3.2. Electromechanical Tests. The electromechanical tests were carried out for analyzing the strain sensitivity of the bricks and evaluate their monitoring performance as strain sensors. All the samples were subjected to step loads: the electrical resistance was obtained through the electrical measurements between two conductive electrodes (both stainless steel and copper ones), while the actual strain was measured using traditional strain gauges, by averaging their results. The calculation of the gauge factor (GF) of all the samples permits to identify the optimal amount of added fillers to obtain the most performing brick sensor. It was obtained through the following equation:

$$
\mathrm{GF}=-\frac{\Delta R / R_{0}}{\Delta \varepsilon},
$$

where $\Delta R$ is the incremental variation in electrical resistance, $R_{0}$ the unstrained electrical resistance, and $\varepsilon$ the axial strain (positive in compression). In plate electrodes, part of the change in electrical resistance with increasing compression load could be attributable to a change in contact resistance, at low values of compression. The detailed analysis of this aspect goes however beyond the purposes of the present study where a preload is applied to mitigate this phenomenon. The data acquisition system and the voltage applying methodology were the same with the electrical tests. The data acquisition system for the strain gauges was model PXIe-4330, 8 channels, 24-bit resolution, $25 \mathrm{kHz}$ of maximum sampling rate, and antialiasing filters. The step loads were applied using a LAUMAS CL press with a $2000 \mathrm{~kg}$ load cell. The load step was between 500 and $1200 \mathrm{~N}$. Both clay bricks with stainless steel fibers and titania particles were investigated, in order to compare their electrical behavior and their sensitivity with different amounts of inclusions.

Figure 5 shows the setup for the electrical and electromechanical tests. The photograph shows the complete measurement setup during an electrical test. The signal generator (Figure 5(a)) provided the voltage square wave, and the data acquisition system recorded the current flowing between the electrodes. Figure 5(b) also illustrates the biphasic measurement procedure, while Figure 5(c) shows the cyclical load history provided by the press.

\section{Results and Discussion}

4.1. Electrical Tests. Figure 6 shows the outputs of the electrical tests on brick samples doped with different amounts of stainless steel microfibers. Figure 6(a) depicts the comparison between the results obtained using embedded steel wire electrodes and external copper plate electrodes: the external plate electrodes provided an electrical resistance variation which decreased with the increase of the amount of conductive fillers. On the contrary, when using embedded stainless steel electrodes, an unexpected increase in electrical resistance variation with increasing amount of steel microfibers was evidenced. Such effect was probably due to the contact resistance between the wire electrodes and the brick matrix with steel fibers. Indeed, the internal electrodes represented points of discontinuity in the material, and during the burning, such discontinuity originated internal local macro- and microporosity which affected the contact between clay and metallic electrodes. So, the increase of the electrical resistance variation with the increase of the amount of added conductive 


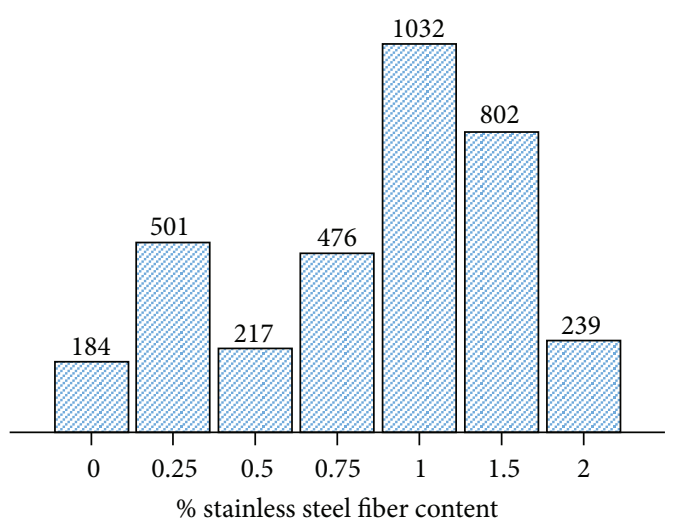

(a)

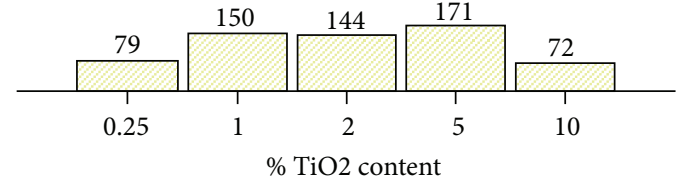

(b)

FIGURE 7: Gauge factor values of smart bricks as a function of (a) stainless steel fiber content (with copper plate electrodes) and (b) content of titania (with stainless steel embedded point electrodes).

fillers was probably due to the higher interference effect of the embedded electrodes on the composite with higher presence of solid inclusions. Figure 6(b) shows the average electrical resistance in bricks with increasing amounts of conductive fillers and the observed variability for the different measurements, in the form of error bars. The results show that the presence of the conductive fillers in the clay determines a smaller variability of the results, that is, a higher repeatability of the measurements.

4.2. Electromechanical Tests. The gauge factor was calculated from results obtained with bricks lying in the horizontal configuration, for both typologies of inclusions and by increasing their amounts. Figure 7(a) shows that, in the case of bricks with stainless steel microfibers with copper plate electrodes, the sample with the highest sensitivity is the one with $1 \%$ of conductive additions, with a notable GF of 1032. The variability of the measured GF with different amounts of fiber contents is probably due to the possible occurrence of local concentrations of fibers due to the bond among them. For comparative purposes, Figure 7(b) reports the gauge factors of bricks doped with titania and embedded electrodes as used in the previous work [12]: the brick with $5 \%$ of filler shows the highest value of the gauge factor. The comparison of such results allows to conclude that the bricks with steel microfibers manifest the highest sensitivity with a much lower amount of filler in comparison to smart bricks doped with titania. This is conceivably due to the peculiar aspect ratio of the inclusions and to the higher electrical conductivity of stainless steel microfibers with respect to titania. Gauge factors of bricks proposed in this work are also seen to be much higher than those of bricks with titania. However, this result cannot be generalized due to the different configurations of electrodes (copper plate electrodes for bricks made with stainless steel microfibers and stainless steel point electrodes for bricks doped with titania and proposed in the previous work). As a final investigation on the strainsensing properties of smart bricks doped with stainless steel microfibers, Figure 8 depicts the time histories of measured strain and of corresponding normalized variation of electrical resistance, during an electromechanical test on samples with plate electrodes. Particularly, Figure 8(a) refers to a normal brick, while Figure 8 (b) refers to the brick with $1 \%$ of steel microfibers. The graphs show that the presence of the fillers increases the stiffness of the bricks, producing a lower strain under the same applied load. Furthermore, the sensitivity of the doped brick is notably higher than that of the normal one, and also the quality of the output signal appears to be much improved with the addition of microfibers.

\section{Conclusions}

This paper presented a new formulation for smart clay bricks, a novel sensing technology recently proposed by the authors for structural health monitoring of masonry structures, based on the use of stainless steel microfibers as conductive fillers and superficial horizontal copper plates (applied postbaking of clay) as electrodes. Traditional clay bricks were doped with different amounts of fillers, from $0 \%$ to $2 \%$. Such peculiar fillers possessed a great resistance to high temperature, as demonstrated through the thermogravimetric tests. This characteristic was essential to ensure brick's conductivity and piezoresistivity after heating at $900^{\circ} \mathrm{C}$ that is reached during their production process. Electrical tests carried out with different electrodes' configurations showed that horizontal plate copper electrodes, placed on the top and bottom external surfaces of the bricks, exhibited more reliable and repeatable results in comparison to those obtained using embedded electrodes. Furthermore, higher amounts of fillers dispersed in the clay matrix produced samples with a better homogeneity. Electromechanical tests were aimed at investigating the sensing capabilities of the doped bricks. A biphasic methodology was used to apply the voltage to the samples, removing the polarization effect which usually affects traditional DC electrical measurements. The sample with $1 \%$ of stainless steel microfibers was the most sensitive sample among those with different contents of microfibers or titania. Overall, the presented results demonstrate that the new typology of clay-based smart bricks is promising for structural health monitoring application to 

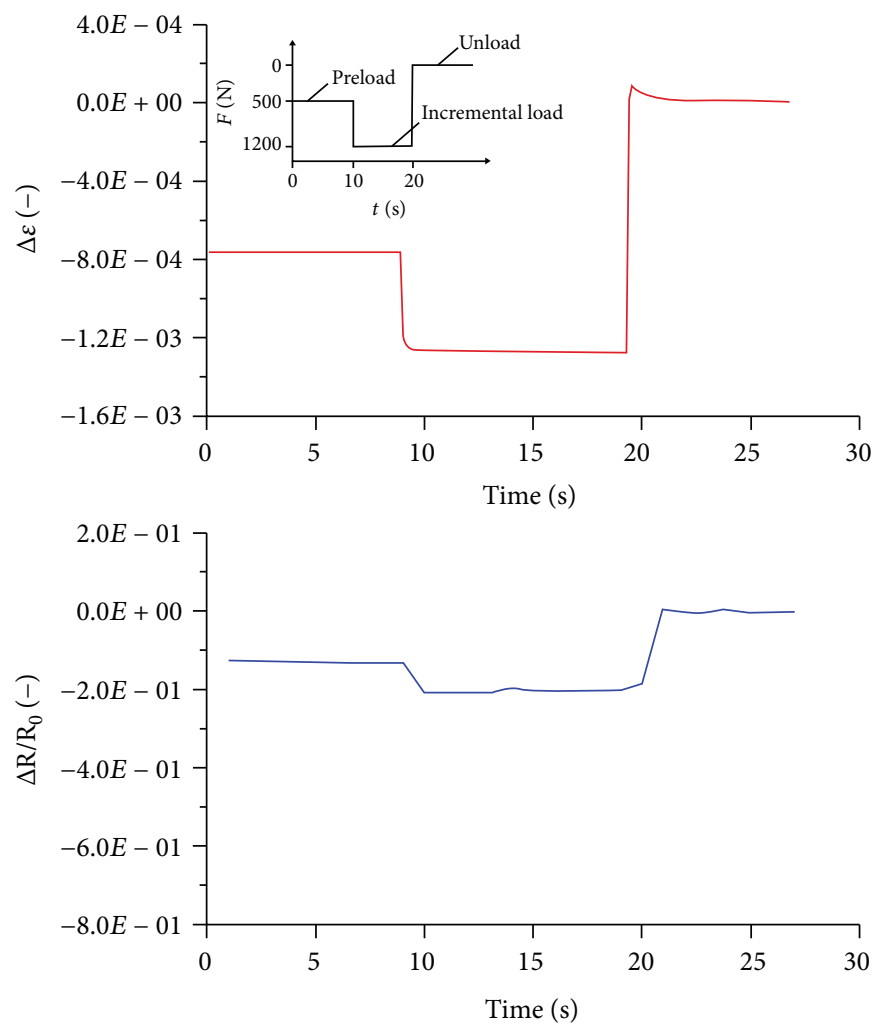

(a)
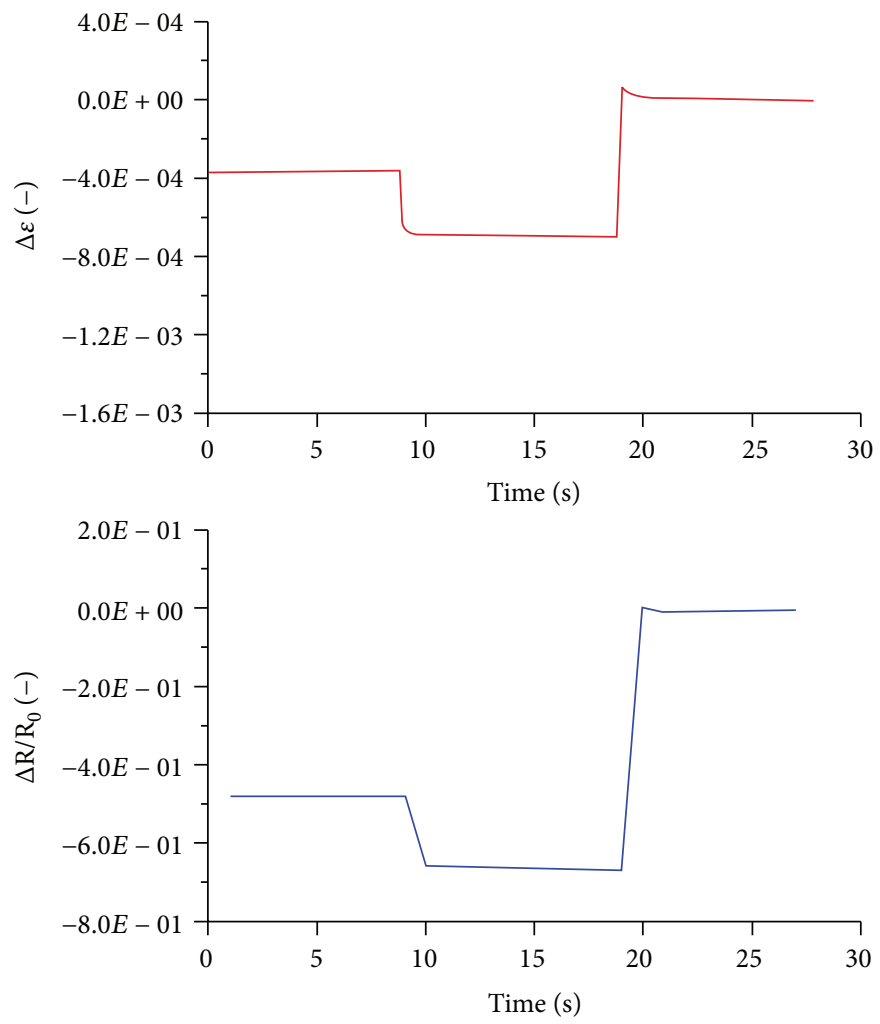

(b)

Figure 8: Time histories of the fractional change in electrical resistance, $\Delta R / R_{0}$, and of the applied strain, $\Delta \varepsilon$, obtained from the electromechanical tests where $R_{0}$ is the electrical resistance value with a preload of $0.5 \mathrm{kN}$, in the case of (a) load applied on clay brick with $0.00 \%$ of stainless steel microfibers; (b) load applied on clay brick with $1.00 \%$ of stainless steel microfibers. 
masonry structures, outperforming the previously proposed smart bricks incorporating titanium dioxide particles and using embedded point electrodes. Their applications may potentially include structural health monitoring of new and existing masonry structures, including historical and monumental ones, which is also made possible by the circumstance that the proposed smart bricks are aesthetically similar to normal ones.

\section{Data Availability}

The data used to support the findings of this study are included within the article.

\section{Conflicts of Interest}

The authors declare that there is no conflict of interest regarding the publication of this paper.

\section{Acknowledgments}

This work was supported by the Italian Ministry of Education, University and Research (MIUR) through the funded project of Relevant National Interest "SMART-BRICK: Novel strain-sensing nanocomposite clay brick enabling selfmonitoring masonry structures" (Protocol no. 2015MS5L27).

\section{References}

[1] J. M. W. Brownjohn, "Structural health monitoring of civil infrastructure," Philosophical Transactions of the Royal Society A: Mathematical, Physical and Engineering Sciences, vol. 365, no. 1851, pp. 589-622, 2007.

[2] S. Laflamme, L. Cao, E. Chatzi, and F. Ubertini, "Damage detection and localization from dense network of strain sensors," Shock and Vibration, vol. 2016, Article ID 2562949, 13 pages, 2016.

[3] F. Magalhaes, A. Cunha, and E. Caetano, "Vibration based structural health monitoring of an arch bridge: from automated OMA to damage detection," Mechanical Systems and Signal Processing, vol. 28, pp. 212-228, 2012.

[4] B. Han, X. Yu, and J. Ou, "Multifunctional and smart nanotube reinforced cement-based materials," in Nanotechnology in Civil Infrastructure. A Paradigm shift, K. Gipalakrishnan, B. Birgisson, P. Taylor, and N. Attoh-Okine, Eds., pp. 1-48, Springer, 2011.

[5] K. J. Loh, T.-C. Hou, J. P. Lynch, and N. A. Kotov, "Carbon nanotube sensing skins for spatial strain and impact damage identification," Journal of Nondestructive Evaluation, vol. 28, no. 1, pp. 9-25, 2009.

[6] J. Wu, C. Song, H. S. Saleem, A. Downey, and S. Laflamme, "Network of flexible capacitive strain gauges for the reconstruction of surface strain," Measurement Science and Technology, vol. 26, no. 5, article 055103, 2015.

[7] D. D. L. Chung, "Piezoresistive cement-based materials for strain sensing," Journal of Intelligent Material Systems and Structures, vol. 13, no. 9, pp. 599-609, 2002.

[8] Y. Ding, Z. Chen, Z. Han, Y. Zhang, and F. Pacheco-Torgal, "Nano-carbon black and carbon fiber as conductive materials for the diagnosing of the damage of concrete beam," Construction and Building Materials, vol. 43, pp. 233-241, 2013.
[9] B. Han, S. Ding, and X. Yu, "Intrinsic self-sensing concrete and structures: a review," Measurement, vol. 59, pp. 110-128, 2015.

[10] L. Coppola, A. Buoso, and F. Corazza, "Electrical properties of carbon nanotubes cement composites for monitoring stress conditions in concrete structures," Applied Mechanics and Materials, vol. 82, pp. 118-123, 2011.

[11] A. D’Alessandro, F. Ubertini, E. García-Macías et al., "Static and dynamic strain monitoring of reinforced concrete components through embedded carbon nanotube cement-based sensors," Shock and Vibration, vol. 2017, Article ID 3648403, 11 pages, 2017.

[12] A. Downey, A. D’Alessandro, S. Laflamme, and F. Ubertini, "Smart bricks for strain sensing and crack detection in masonry structures," Smart Materials and Structures, vol. 27, no. 1, article 015009, 2018.

[13] A. Downey, A. D’Alessandro, F. Ubertini, S. Laflamme, and R. Geiger, "Biphasic DC measurement approach for enhanced measurement stability and multi-channel sampling of selfsensing multi-functional structural materials doped with carbon-based additives," Smart Materials and Structures, vol. 26, no. 6, p. 065008, 2017. 


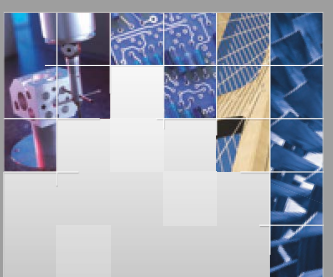

\section{Enfincering}
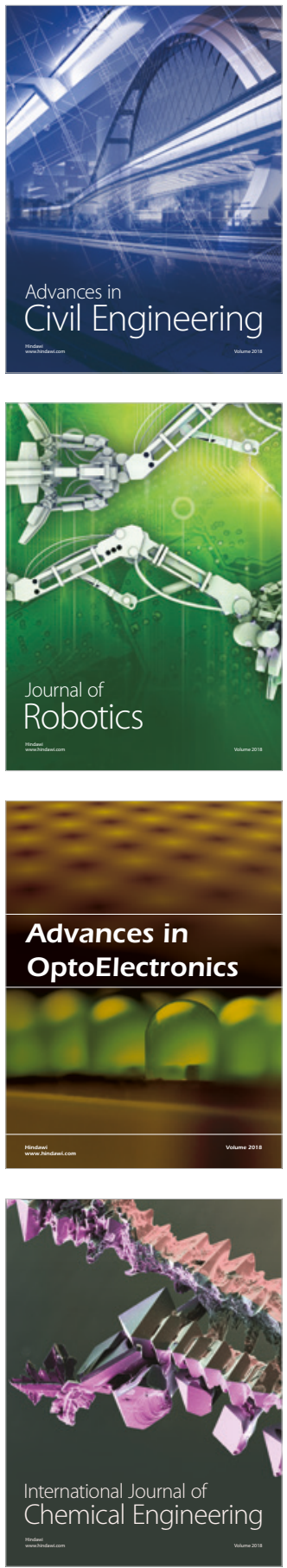

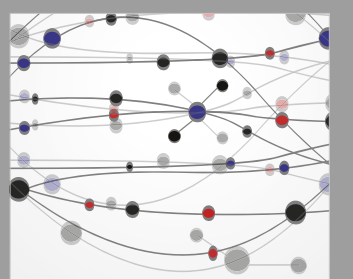

\section{Rotating \\ Machinery}

The Scientific World Journal

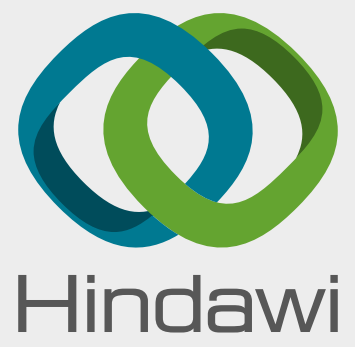

Submit your manuscripts at

www.hindawi.com
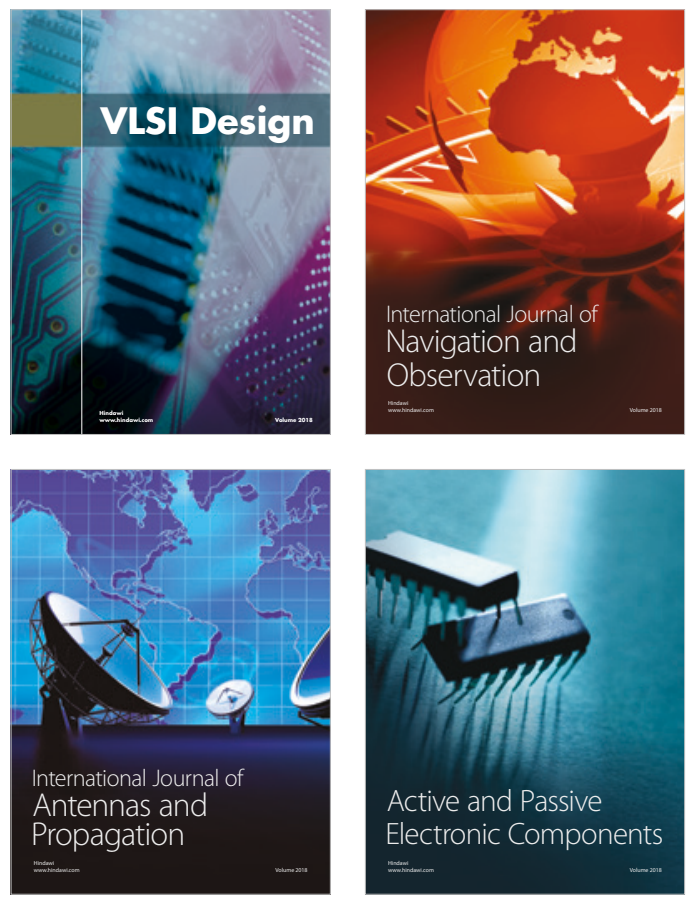
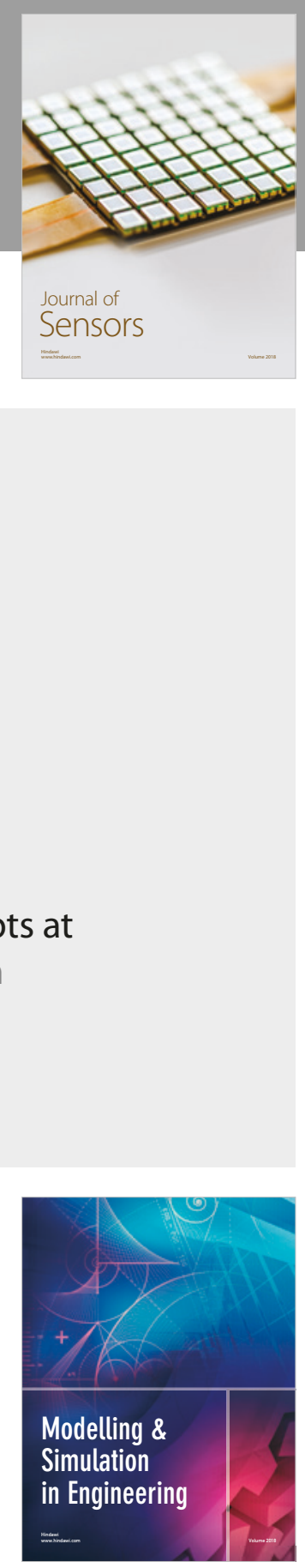

\section{Advances \\ Multimedia}
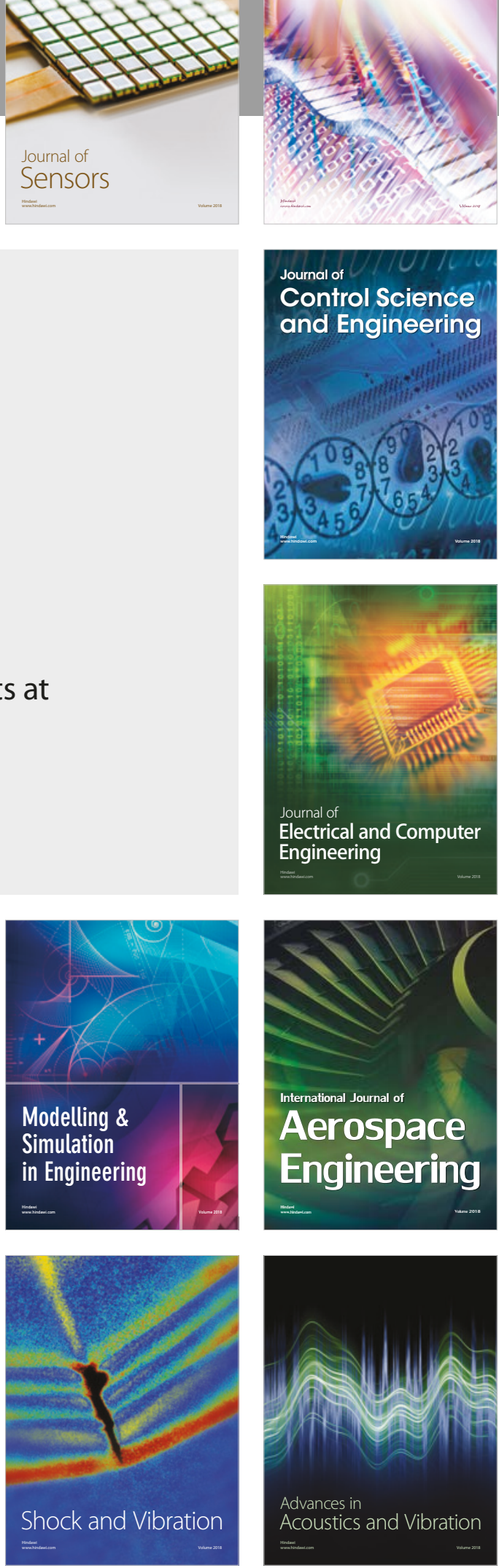